\title{
Limit cycles for a class of second order differential equations.
}

\author{
Jaume Llibre $^{\mathrm{a}}$, Perez-Chavela Ernesto ${ }^{\mathrm{b}, *}$ \\ ${ }^{a}$ Departament de Matemàtiques, Universitat Autònoma de Barcelona, 08193 Bellaterra, Barcelona, \\ Catalonia, Spain \\ ${ }^{b}$ Departamento de Matemáticas, UAM-Iztapalapa, Apdo. Postal 55-534, 09340, México, D.F., México
}

\begin{abstract}
We study the limit cycles of a wide class of second order differential equations, which can be seen as a particular perturbation of the harmonic oscillator. In particular, by choosing adequately the perturbed function we show, using the averaging theory, that it is possible to obtain as many limit cycles as we want.
\end{abstract}

Keywords: Limit cycle, second order differential equation, averaging theory.

\section{Introduction and statement of the main results}

The second order differential equations arise in many areas of science and technology essentially, but in the last years many important applications in social science, economics and business administration have raised, as well as plenty of applications in physics, chemistry, biology and the engineries. Nevertheless, in general is impossible to determine the solution of a second order differential equation in terms of explicit functions, so we look for properties of these kind of equations which can be obtained without solving it. The problem is more relevant in perturbation theory, where the periodic orbits play a main role. For more information about the study of the periodic orbits of second order differential equations see for instance the references $[6,7,8,14,15]$, and the ones quoted there.

It is well known that all solutions of the harmonic oscillator are periodic with the same period, i.e. the origin of this system is an isochronous center. When we take a perturbation of the harmonic oscillator, which periodic orbits persist? which periodic orbits generate limit cycles? In this paper we ask these questions for a wide class of second order differential equations, which are essentially a perturbation of the harmonic oscillator. More precisely, we study equations of the form

$$
\ddot{x}=-\left(1+\varepsilon \kappa \cos ^{2} t\right) x+\varepsilon f(t, x, \dot{x}),
$$

where $\varepsilon$ is a small parameter, $\kappa$ is a real parameter and the function $f(t, x, \dot{x})$ is at least of class $C^{2}$ and $2 \pi$-periodic in the variable $t$ in order to apply the averaging theory [9].

\footnotetext{
*Corresponding author. Tel.: +52 555804 4654; fax: +52 5558044653

Email addresses: jllibre@mat.uab.cat (Jaume Llibre), epc@xanum.uam.mx (Perez-Chavela Ernesto
} ) 
We would like to mention the Ermakov systems, which have a long history in sciences and many important applications in Physics. In the last years many people have retook their study, see for instance $[2,4,5]$. Since the classical Ermakov system has the form

$$
\ddot{x}=-\omega^{2}(t) x+\frac{1}{x^{3}} G(x) .
$$

The class of second order differential equations (1) contains the subclas of Ermakov systems given by the functions $G(x)=\varepsilon x^{3} f(x)$ and $\omega^{2}(t)=1+\varepsilon \kappa \cos ^{2} t$.

The first goal of this paper is provide a general result which, for $\varepsilon$ sufficiently small, allows to study the periodic orbits of the second differential equation (1) for an arbitrary function $f(t, x, \dot{x})$, see the next Theorem 1 . After, we shall compute explicitly the periodic orbits of such second order differential equations for some explicit functions $f(t, x, \dot{x})$, see the next Propositions, 2 and 3.

For stating our results we need some definitions. Thus we define the functions

$$
\begin{aligned}
h_{1}(s, r, a)= & \kappa r \cos s \cos ^{2}(a+s) \sin (a+s)- \\
& f(a+s, r \cos s,-r \sin s) \sin (a+s), \\
h_{2}(s, r, a)=\quad-\kappa r \cos s \cos ^{3}(a+s)+ & f(a+s, r \cos s,-r \sin s) \cos (a+s),
\end{aligned}
$$

and

$$
\mathrm{f}_{j}(r, a)=\frac{1}{2 \pi} \int_{0}^{2 \pi} h_{j}(s, r, a) d s \quad \text { for } \quad j=1,2 .
$$

We say that $\left(r_{0}, a_{0}\right)$ is a simple solution of system

$$
\mathrm{f}_{1}(r, a)=0, \quad \mathrm{f}_{2}(r, a)=0
$$

if the determinant $\left(\frac{\partial\left(\mathrm{f}_{1}, \mathrm{f}_{2}\right)}{\partial(r, a)}\right)\left(r_{0}, a_{0}\right) \neq 0$.

We recall that a limit cycle of the second order differential equation (1) is a periodic orbit isolated in the set of all periodic orbits of (1).

Now we can state the main result of this paper.

Theorem 1. Let $\left(r_{0}, a_{0}\right)$ be a simple solution of system (2). Then for $\varepsilon \neq 0$ sufficiently small the second order differential equation (1) has a limit cycle $x_{\varepsilon}(t)$, such that when $\varepsilon \rightarrow 0$ it tends to the $2 \pi$-periodic solution $r_{0} \cos \left(a_{0}-t\right)$ of system (1) with $\varepsilon=0$.

Theorem 1 will be proved in section 3 .

This theorem allow to determine the existence of limit cycles of the second order differential equation (1) with $\varepsilon \neq 0$ sufficiently small, and the periodic orbits of the harmonic oscillator (1) with $\varepsilon=0$ from which they bifurcate. To show the powerful of Theorem 1 we give some applications of it, we obtain that by choosing conveniently the perturbed function, equation (1) can have one, or as many limit cycles as we want. The following propositions, whose proofs will be given in section 4 , show these different possibilities. 
Proposition 2. For $\varepsilon \neq 0$ sufficiently small and for all $\kappa>0$ the second order differential equation (1) with $f(t, x, \dot{x})=\cos t$ has a unique limit cycle $x_{\varepsilon}(t)$ such that $x_{\varepsilon}(t) \rightarrow$ $\frac{4}{3 \kappa} \cos t$ as $\varepsilon \rightarrow 0$.

Proposition 3. For $\varepsilon \neq 0$ sufficiently small and $\kappa \in[0,1]$, the second order differential equation (1) with $f(t, x, \dot{x})=\sin x$ can have as many limit cycles as we want.

\section{A basic result from averaging theory}

In order to have a self contained paper, in this section we present the basic results from the averaging theory that are necessary for proving the main result of this paper, i.e Theorem 1.

We considerer a differential system of the form

$$
\dot{\mathbf{x}}(t)=F_{0}(t, \mathbf{x})+\varepsilon F_{1}(t, \mathbf{x})+\varepsilon^{2} F_{2}(t, \mathbf{x}, \varepsilon),
$$

where the functions $F_{0}, F_{1}: \mathbb{R} \times \Omega \rightarrow \mathbb{R}^{n}$ and $F_{2}: \mathbb{R} \times \Omega \times\left(-\varepsilon_{0}, \varepsilon_{0}\right) \rightarrow \mathbb{R}^{n}$ are of class $C^{2}$ in $\Omega$ and $T$-periodic in the variable $t, \Omega$ is an open subset of $\mathbb{R}^{n}$. When $\varepsilon=0$ we get the unperturbed system

$$
\dot{\mathbf{x}}(t)=F_{0}(t, \mathbf{x}),
$$

we assume that the above unperturbed system has an open subset of $\Omega$ fulfilled of periodic solutions. For $\varepsilon$ sufficiently small is possible obtain periodic solutions of system (3) by using the averaging theory.

Let $\mathbf{x}(t, \mathbf{z}, \varepsilon)$ be the solution of system (3) such that $\mathbf{x}(0, \mathbf{z}, \varepsilon)=\mathbf{z}$. The variational equation of the unperturbed system (4) along the periodic solution $\mathbf{x}(t, \mathbf{z}, 0)$ is

$$
y^{\prime}=D_{\mathbf{x}} F_{0}(t, \mathbf{x}(t, \mathbf{z}, 0)) y .
$$

where $y$ is a $n \times n$ matrix. Let $M_{\mathbf{z}}(t)$ be a fundamental matrix of the linear differential system (5). We assume that $\Omega$ contains an open subset $W$ formed only by periodic orbits, all of them having the same period $T$, i.e. $W$ is an isochronous set of system (3). Then we have the following result.

Theorem 4. Assume that $\Omega$ contains an open and bounded set $W$ such that $C l(W) \subset \Omega$ and for each $\boldsymbol{z} \in \mathrm{Cl}(W)$, the solution $x(t, \boldsymbol{z}, 0)$ is $T$-periodic. Let $F: W \rightarrow \mathbb{R}^{2}$ be the function defined by

$$
F(z)=\frac{1}{T} \int_{0}^{T} M_{z}^{-1}(t) F_{1}(t, \boldsymbol{x}(t, \boldsymbol{z}, 0)) d t .
$$

If there exist $\boldsymbol{p} \in W$ with $F(\boldsymbol{p})=0$ and the determinant of $(d F / d \boldsymbol{z})(\boldsymbol{p}) \neq 0$, then there exists a T-periodic solution $\phi(t, \varepsilon)$ of system (3) such that $\phi(0, \varepsilon) \rightarrow \boldsymbol{p}$ as $\varepsilon \rightarrow 0$.

For a shorter proof of Theorem 4 see Corollary 1 of [3]. In fact this result goes back to Malkin [12] and Roseau [13]. For an application of Theorem 4 to Hamiltonian systems in a more general context you can see [10]. 


\section{Proof of Theorem 1}

For proving Theorem 1 we shall use Theorem 4. In what follows we will use the notation introduced in section 2 .

Proof of Theorem 1. The second order differential equation (1) can be written as the first order differential system

$$
\begin{aligned}
& \dot{x}=v, \\
& \dot{v}=-x+\varepsilon\left(-\kappa x \cos ^{2} t+f(t, x, v)\right)=-x+\varepsilon f_{1}(t, x, v) .
\end{aligned}
$$

This system can be written in the vectorial form

$$
\dot{\mathbf{x}}=F_{0}(t, x, v)+\varepsilon F_{1}(t, x, v),
$$

where

$$
\mathbf{x}=\left(\begin{array}{l}
x \\
v
\end{array}\right), \quad F_{0}(t, x, v)=\left(\begin{array}{c}
v \\
-x
\end{array}\right) \quad \text { and } \quad F_{1}(t, x, v)=\left(\begin{array}{c}
0 \\
f_{1}(t, x, v)
\end{array}\right) .
$$

The unperturbed part of system (7) is

$$
\begin{aligned}
& \dot{x}=v, \\
& \dot{v}=-x,
\end{aligned}
$$

it corresponds to the simple harmonic oscillator whose solution is

$$
\begin{aligned}
& x(t)=x_{0} \cos t+v_{0} \sin t, \\
& v(t)=-x_{0} \sin t+v_{0} \cos t .
\end{aligned}
$$

We observe that for any initial condition $\left(x_{0}, v_{0}\right) \in \mathbb{R}^{2} \backslash\{(0,0)\}$, the corresponding solution is $2 \pi$-periodic and can be write as

$$
x(t)=r \cos (a-t), \quad v(t)=r \sin (a-t),
$$

where $r>0$ and $a$ are constants which can be written in terms of the initial conditions $\left(x_{0}, v_{0}\right)$. We say that the origin of system (9) is an isochronous center.

Let $\mathbf{x}(t, \mathbf{z}, \varepsilon)$ be the solution of system (7) such that $\mathbf{x}(0, \mathbf{z}, \varepsilon)=\mathbf{z}=(r, a)$. The variational equation of the unperturbed system (9) along the periodic solution $\mathbf{x}(t, \mathbf{z}, 0)$ is

$$
y^{\prime}=D_{\mathbf{x}} F_{0}(t, \mathbf{x}(t, \mathbf{z}, 0)) y,
$$

where $y$ is a $2 \times 2$ matrix. Let $M(t)$ be a fundamental matrix of the linear differential system (11), it takes the simple form

$$
M(t)=\left(\begin{array}{cc}
\cos t & \sin t \\
-\sin t & \cos t
\end{array}\right) .
$$

Now, evaluating the function $f_{1}(t, x, v)$ along the solution (10) of the unperturbed system we obtain the function

$$
h(t, r, a)=-\kappa r \cos (a-t) \cos ^{2} t+f(t, r \cos (a-t), r \sin (a-t)) .
$$


Then we have

$$
\left.M^{-1}(t) F_{1}(t, \mathbf{x}(t, \mathbf{z}))\right)=\left(\begin{array}{c}
-h(t, r, a) \sin t \\
h(t, r, a) \cos t
\end{array}\right) .
$$

Doing the change of variable $t \rightarrow a+s$ the above expression becomes

$$
\left(\begin{array}{c}
\kappa r \cos s \cos ^{2}(a+s) \sin (a+s)-f(a+s, r \cos s,-r \sin s) \sin (a+s) \\
-\kappa r \cos s \cos ^{3}(a+s)+f(a+s, r \cos s,-r \sin s) \cos (a+s)
\end{array}\right) .
$$

We observe that the first part in the two previous expressions does not depend of the function $f(a+s, r \cos s,-r \sin s)$.

Let

$$
\left.\mathrm{F}(\mathbf{z})=\left(\begin{array}{c}
\mathrm{f}_{1}(r, a) \\
\mathrm{f}_{2}(r, a)
\end{array}\right)=\frac{1}{2 \pi} \int_{0}^{2 \pi} M^{-1}(t) F_{1}(t, \mathbf{x}(t, \mathbf{z}))\right) d t .
$$

Let $W \neq \emptyset$ be an open and bounded subset of $\mathbb{R}^{2}$, formed only by periodic orbits, all of them having the same period $2 \pi$, that is $W$ is a isochronous set for system (9).

Let $\left(r_{0}, a_{0}\right) \in W$ a simple solution of the system

$$
\mathrm{f}_{1}(r, a)=0, \quad \mathrm{f}_{2}(r, a)=0,
$$

that is determinant $\left(\frac{\partial\left(\mathrm{f}_{1}, \mathrm{f}_{2}\right)}{\partial(r, a)}\right)\left(r_{0}, a_{0}\right) \neq 0$.

With all the above we have verified the hypotheses of Theorem 4 , then the proof of Theorem 1 follows from it.

\section{Proof of the propositions}

In this section we present the proof of the propositions stated in section 1 , showing that depending on the function $f(t, x, \dot{x})$ in $(1)$, it is possible to obtain as many limit cycles as we want. Again we will use the notations introduced in section 2 and in the proof of Theorem 1 of section 3 .

Proof of Proposition 2. Taking $f(t, x, v)=\cos t$, the second order differential equation (1) becomes

$$
\ddot{x}=-\left(1+\kappa \varepsilon \cos ^{2} t\right) x+\varepsilon \cos t .
$$

By straightforward computations the value of the integral (12) for the above $f(t, x, v)$ at the initial condition $(r, a)$ is

$$
\mathrm{F}(r, a)=\left(\begin{array}{c}
\kappa r \sin a / 8 \\
(4-3 \kappa r \cos a) / 8
\end{array}\right)
$$

which has a unique zero for $r>0$ given by $(r, a)=(4 /(3 \kappa), 0)$ with $\kappa>0$. The determinant of $(d \mathrm{~F} / d \mathbf{z})(4 /(3 \kappa), 0)=\frac{1}{16 \kappa} \neq 0$. Then applying Theorem 1 , for $\kappa>0$ we can assure the existence of a unique $2 \pi$-periodic solution $\mathbf{x}_{\varepsilon}(t)$ of system (14) such that

$$
x_{\varepsilon}(t) \rightarrow \frac{4}{3 \kappa} \cos t \quad \text { as } \quad \varepsilon \rightarrow 0 .
$$

So Proposition 2 is proved. 
(a)

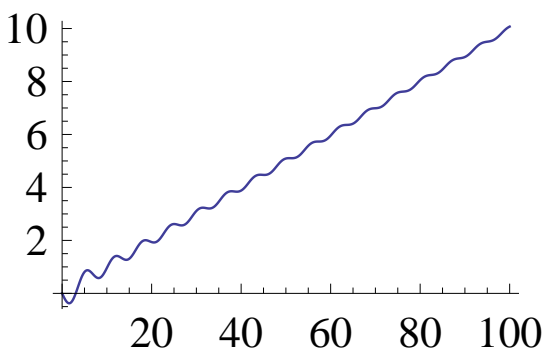

(c)

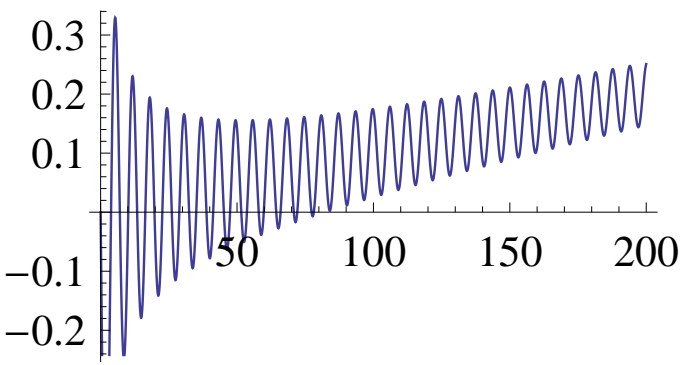

(b)

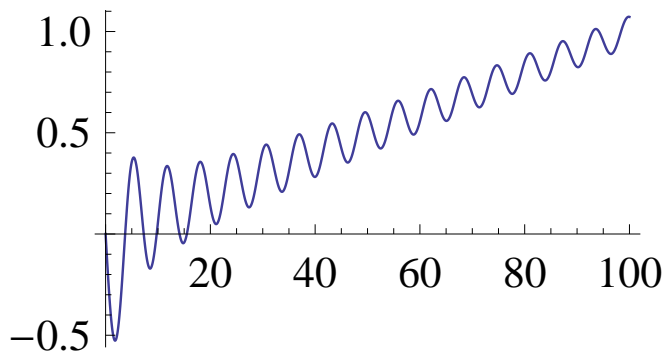

(d)

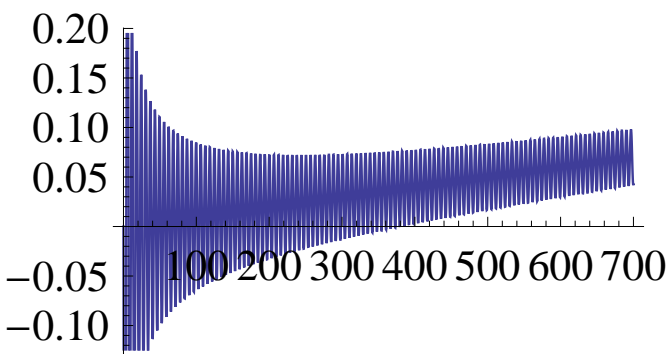

Figure 1: Graphs of the functions $\kappa r-J_{1}(r) \sin a^{*}$ for (a) $\kappa=0.1$; (b) $\kappa=0.01$; (c) $\kappa=0.001$ and $(\mathrm{d}) \kappa=0.0001$.

Proof of Proposition 3. In this case the function $f(t, x, v)=\sin x$ depends only on $x$. So we must analyze the differential system

$$
\begin{aligned}
\dot{x} & =v, \\
\dot{v} & =-\left(1+\varepsilon \kappa \cos ^{2} t\right) x+\varepsilon \sin x .
\end{aligned}
$$

Evaluating the function $f_{1}(t, x, v)=-x \kappa \cos ^{2} t+\sin x$ along the $2 \pi$-periodic solutions (10) of the unperturbed system, we obtain

$$
h(t, r, a)=-\kappa r \cos (a-t) \cos ^{2} t+\sin (r \cos (a-t)),
$$

therefore $M^{-1}(t) F_{1}(t, \mathbf{x}(t,(r, a)))$ becomes

$$
\left(\begin{array}{c}
-\sin t\left(-\kappa r \cos (a-t) \cos ^{2} t+\sin (r \cos (a-t))\right) \\
\cos t\left(-\kappa r \cos (a-t) \cos ^{2} t+\sin (r \cos (a-t))\right)
\end{array}\right) .
$$

Now doing the change of variable $t \rightarrow a+s$ we have

$$
\left(\begin{array}{c}
\kappa r \cos s \cos ^{2}(a+s) \sin (a+s)-\sin (a+s) \sin (r \cos s) \\
-\kappa r \cos s \cos ^{3}(a+s)+\cos (a+s) \sin (r \cos s)
\end{array}\right)
$$

Finally integrating these expressions with respect to the variable $s$ from 0 to $2 \pi$, dividing by $2 \pi$ and assuming that $r>0$, we obtain

$$
\left(\begin{array}{c}
\mathrm{f}_{1}(r, a) \\
\mathrm{f}_{2}(r, a)
\end{array}\right)=\frac{1}{8}\left(\begin{array}{c}
\kappa r-J_{1}(r) \sin a \\
-3 \kappa r+8 J_{1}(r) \cos a, \\
6
\end{array}\right)
$$


where $J_{1}(r)$ is the Bessel function of the first kind, for a precise definition see [1].

The system $\mathrm{f}_{1}(r, a)=\mathrm{f}_{2}(r, a)=0$ is equivalent to the system

$$
8 \cos a=3 \sin a, \quad \kappa r-J_{1}(r) \sin a=0 .
$$

Taking $a=a^{*}$ one of the values of $\arctan (8 / 3)$, the previous system reduces to the unique equation

$$
\kappa r-J_{1}(r) \sin a^{*}=0 .
$$

Then the simple solutions of the system $\mathrm{f}_{1}(r, a)=\mathrm{f}_{2}(r, a)=0$ correspond to the simple solutions of equation (15), which depend on the value of the parameter $\kappa$. For instance, for $\kappa=1$ the above system has exactly one simple zero at $r=0$, for $\kappa=0$ it corresponds to the Bessel function which has infinitely many simple zeroes. It is not difficult to verify that for $\kappa \in(0,1)$, we can have as many simple solutions as we want. In Figure 1 we have plot the graphs of some functions $\kappa r-J_{1}(r) \sin a^{*}$ changing the value of $\kappa$. The proof of Proposition 3 is complete.

\section{Aknowledgments}

The first author is partially supported by a MCYT/FEDER grant MTM2008-03437, by a CIRIT grant number 2009SGR-0410 and by ICREA Academia. The second author is partially supported by CONACYT México, grant No. 128790 .

\section{References}

[1] M. Abramowitz and I. Stegun, Handbook of Mathematical Functions with Formulas, Graphs, and Mathematical Tables, National Institute of Standards and Technology, Dover Publications, 1964

[2] C. Athorne, C. Rogers, U. Ramgulam and A. Osbaldestin, On linearization of the Ermakov system, Physics Letters A 143 (1990), 207-212.

[3] A.Buica, J.P. Francoise And J. Llibre, Periodic solutions of non-linear periodic differential systems with a small parameter, Communications on Pure and Applied Analysis 6 (2007), 103-111.

[4] J. F. Cariñena, J. DE LuCAs And M. F. RañAda, Recent Applications of the Theory of Lie Systems in Ermakov Systems, Symmetry, Integrability and Geometry: Methods and Applications 4 (2008), 031, 18 pages.

[5] F. HaAs And J. Goedert, Lie point symmetries for reduced Ermakov system, Physics Letters A 332 (2004), 25-34.

[6] X. Li AND Z. ZHANG, Unbounded solutions and periodic solutions for second order differential equations with asymmetric nonlinearity, Proc. Amer. Math. Soc. 135 (2007), 2769-2777.

[7] X. Li AND Z. ZhAng, Periodic solutions for second-order differential equations with a singular nonlinearity, Nonlinear Anal. 69 (2008), 3866-3876.

[8] X. Li AND Z. Zhang, Periodic solutions for some second order differential equations with singularity, Z. Angew. Math. Phys. 59 (2008), 400-415.

[9] J. Llibre, C.A. Buzzi And P.R. DA Silva, 3-dimensional Hopf bifurcation via averaging theory, Discrete and Continuous Dynamical Systems 17 (2007), 17 (2007), 529-540.

[10] J. Llibre And A. Rodrigues, On the periodic orbist of the Hamiltonian Systems, J. Mathematical Physics 51, 042704 (2010).

[11] J. Llibre, J. Yu AND X. Zhang, Limit cycles for a class of third order differential equations, Rocky Mountain J. of Math. 40 (2010), 581-594.

[12] I.G. Malkin, Some problems of the theory of nonlinear oscillations, (Russian) Gosudarstv. Izdat. Tehn.-Teor. Lit., Moscow, 1956.

[13] M. Roseau, Vibrations non linéaires et théorie de la stabilité, (French) Springer Tracts in Natural Philosophy, Vol.8 Springer-Verlag, Berlin-New York, 1966.

[14] K. SchmitT, Periodic solutions of second order equations-a variational approach. The first 60 years of nonlinear analysis of Jean Mawhin, World Sci. Publ., River Edge, NJ, 2004, pp 213-220. 
[15] E. Serra, M. Tarallo and S. Terracini, Subharmonic solutions to second-order differential equations with periodic nonlinearities., Nonlinear Anal. Ser. A: Theory Methods 41 (2000), 649667. 